\title{
The association between low grade inflammation and skin diseases: A cross-sectional survey in the Northern Finland Birth Cohort 1966
}

\author{
S-P. Sinikumpu', L.Huilaja ', J.Auvinen ${ }^{2}$, J. Jokelainen ${ }^{2}$, K.Puukka ${ }^{3}$,A.Ruokonen ${ }^{3}$, M.Timonen, ${ }^{2}$ K.Tasanen' \\ IPEDEGO Research Unit, University of Oulu; Department of Dermatology and Medical Research Center Oulu, Oulu University Hospital P.O.Box 20,90029 Oulu University Hospital, Oulu, Finland \\ ${ }^{2}$ Center for Life Course Health Research, Faculty of medicine University of Oulu and Oulu University Hospital, Oulu, Finland \\ ${ }^{3}$ NordLab Oulu, Medical Research Center Oulu, Oulu University Hospital and Department of Clinical Chemistry, University of Oulu, Finland
}

\section{INTRODUCTION}

- Low grade inflammation is a chronic, subclinical and systemic condition (1), which contributes to the pathogenesis of many noncommunicable diseases, such as atherosclerosis (2) and type 2 diabetes (3).

- Low grade inflammation is detectable via a minor elevation of certain inflammatory markers, such as C-reactive protein (CRP), and it is usually measured by a high sensitivity CRP (hs-CRP) test $(4,5)$.

- Although several skin diseases $(5,6,7)$ have an inflammatory background, the association between skin diseases and systemic inflammation has been studied only in a disease-specific manner.

- By using data from the unique 1966 Northern Finland Birth Cohort 1966 (NFBC1966) we aimed to determine, in an unselected adult population, any association between low grade inflammation and any of the most common skin diseases.

\section{MATERIAL AND METHODS}

- A whole body investigation on 1, 930 adults belonging to a well documented regional population that has been followed since birth in 1966 was performed by dermatologists.

- High sensitive C-reactive protein (hs-CRP) level was measured as a marker of low grade inflammation ( $n=1,906, n=884$ males and $n=1,022$ females).

- Multinomial logistic regression analysis was used to calculate the association between inflammation and selected skin disorders.

\section{RESULTS}

- Hs-CRP was normal (<1 mg/L) in $61.6 \%$, slightly elevated $(1-3 \mathrm{mg} / \mathrm{L})$ in $28.0 \%$ and highly elevated ( $>3 \mathrm{mg} / \mathrm{L}$ ) in $10.4 \%$ of the participants. Median hs-CRP level for females was $0.8 \mathrm{mg} / \mathrm{L}$ and $0.7 \mathrm{mg} / \mathrm{L}$ for males, $(p=0.27)$ (Table I).

- Hs-CRP was higher in participants with atopic eczema $(p=0.03)$, rosacea $(p=$ $0.001)$ and onychomycosis $(p=0.01)$ (Table II)

- Hs-CRP was also higher in study cases who had any skin disease that was classified as severe enough needing further evaluation or treatment, compared to those needing no further care $(p=0.016)$ (Table II).

\section{- After adjustment for confounding factors (smoking, education, physical} activity, systemic disease and the use of contraceptives) atopic eczema (OR 2.2, 95\% Cl 1.2-3.9), onychomycosis (OR 2.0, 1.2-3.2) and rosacea (OR 1.7, 1.12.5) associated with low grade inflammation (Table III).

- After additionally adjusting for body mass index, the risks for atopic eczema (OR 2.4, 1.3-4.6) and onychomycosis (OR 1.9, 1.1-2.0) remained statistically significant (Table III).

\section{CONCLUSIONS}

\section{- Low grade inflammation is present in several skin diseases}

- It is known that the presence of one inflammatory disease exacerbates the risk for others (8). Thus, patients with dermatological conditions such atopic eczema, rosacea or fungal skin infection, may carry a heightened risk for noncommunicable inflammatory diseases such as atherosclerosis, and this should be kept in mind when treating patients with cutaneous disorders.

\section{Contact information
Suvi-Päivikki Sinikumpu, MD, Clinical Research Center Aepartment of
Alistie $5 \mathrm{~A}$
F-90220 Oulu E-mail: suv E-mail: suvi-
paivikki.sinikumpu@oulu.fi}

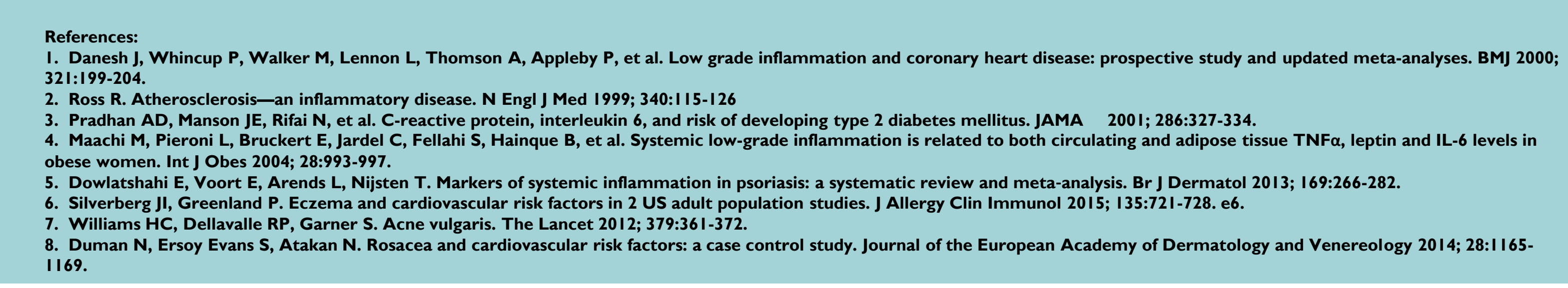

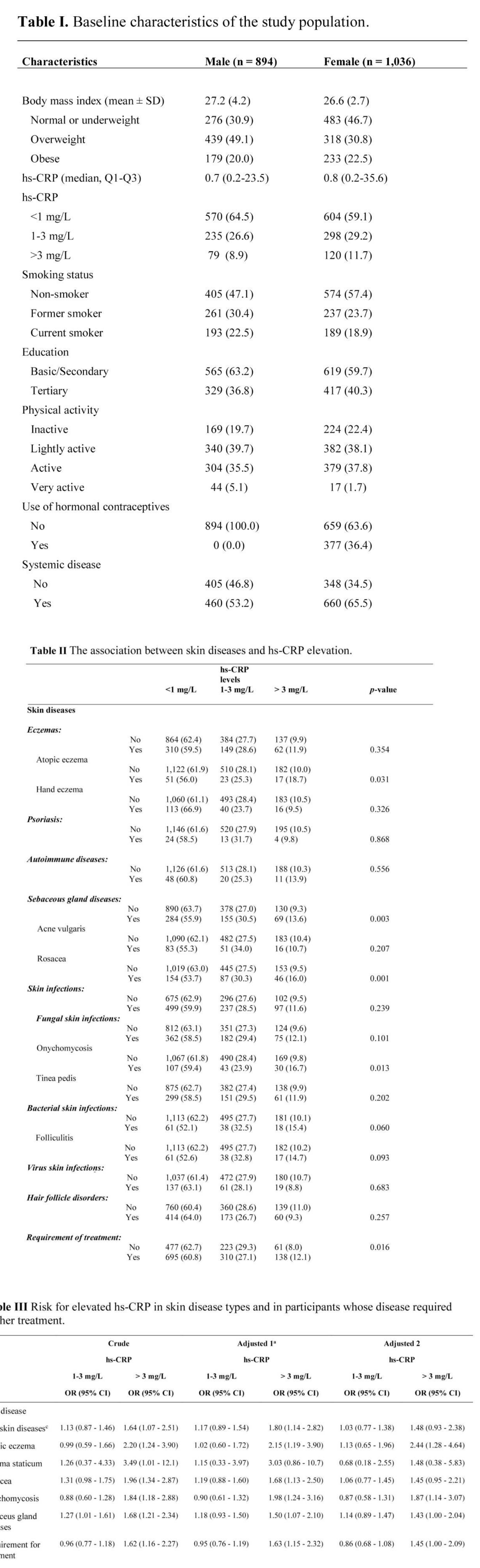

